\title{
Circuit-Switched Wireless Sensor Networks: A Discrete-Time Communication Model for Performance Analysis ${ }^{\dagger}$
}

\author{
Gianluigi Ferrari ${ }^{1,2}$, and Ozan K. Tonguz ${ }^{1}$ \\ ${ }^{1}$ Electrical and Computer Engineering Department, Carnegie Mellon University \\ Pittsburgh, PA, 15213-3890, USA - Phone: 412-268-5991 Fax: 412-268-2860 \\ ${ }^{2}$ Dipartimento di Ingegneria dell'Informazione, University of Parma \\ I-43100, Parma, Italy \\ E-mail: gferrari@cmu.edu,tonguz@ece.cmu.edu
}

\begin{abstract}
In this paper, we consider a novel discrete-time model to analyze the performance of circuit-switched sensor networks. In particular, we assume that a node, after reserving a multi-hop communication route to the desired destination, holds it for a time interval defined as reserved channel utilization interval (RCUI) and utilizes it for an effective channel utilization interval (ECUI). A realistic network communication scenario with inter-node interference (INI) and a reservation-based medium access control (MAC) protocol with finite numbers of (active) routes (FNR) in the network is first considered, and the average interference power is evaluated through a novel combinatorial analysis. Results are presented in terms of effective transport capacity and channel utilization ratio (CUR). In particular, we show that for very low values of the packet generation rate at each node, activation of the maximum possible number of routes guarantees no loss, in terms of effective transport capacity, with respect to an ideal (no INI) scenario. However, this comes at the expense of a very low utilization: in other words, once a multihop route has been reserved, its effective utilization time must be a few orders of magnitude lower than the duration of the reservation interval.
\end{abstract}

\section{INTRODUCTION}

Ad hoc wireless networks represent a new communication paradigm which has been developing over the last years [1], [2]. While ad hoc wireless networks hold the potential to guarantee ubiquitous connectivity, it is still not clear how such networks would work. In fact, most of the existing literature focuses on routing aspects, neglecting fundamental limitations at the physical layer.

In [3]-[6], a particular communication paradigm for wireless sensor networks has been proposed. More precisely, a circuit-switched multi-access communication network is considered, where a source node, in need of communicating with a destination node, first reserves a multi-hop communication route and then transmits its message (the multi-hop route is constituted by a sequence of "relay" nodes which simply carry on the information generated at the source node). This network communication scenario can be analyzed in terms of effective transport capacity, introduced in [6], representing the

\footnotetext{
${ }^{\dagger}$ The Authors were funded in part by Army Research Office (ARO) under Contract No. DAAD19-02-1-0389 and CyLab of Carnegie Mellon University (CMU). Any opinions, findings, and conclusions or recommendations expressed in this publication are those of the authors and do not necessarily reflect the views of the ARO or CyLab of CMU.
}

actual bandwidth-distance product carried by the network. The overall maximum of this quantity corresponds to the transport capacity of the network [7].

From the results in [5], [6], it is possible to deduce that, in order for a circuit-switched wireless sensor network to perform well, a node must not utilize the reserved route for most of the reservation interval. In this paper, we investigate further this aspect. To this end, we introduce an equivalent discrete-time communication model, and we assume that each node, after reserving a multi-hop route to its destination, can hold it for a reserved channel utilization interval (RCUI). The duration of the message to be transmitted, i.e., the duration of the effective channel utilization interval (ECUI), must be lower than the duration of the RCUI. For the sake of analytical simplicity, we will assume that a node transmits its message, within the RCUI, in a randomized way-this simplifies the analysis, but it is possible to show that our conclusions hold also for other transmission strategies inside the RCUI. The channel utilization ratio (CUR) defined as the ratio between the durations of the ECUI and RCUI, will be introduced and evaluated as a meaningful network performance indicator. The introduced discrete-time model allows to perform a novel combinatorial analysis of the inter-node interference (INI).

\section{Conceptual Model}

The underlying assumptions behind the considered sensor network communication model are as follows.

- Peer-to-peer multi-hop circuit switching with disjoint routes is considered (a node can not serve as a relay in more than one route).

- The nodes are static and placed at the vertices of a regular grid.

- The route creation phase is not explicitly taken into account (we assume that the routes are created and we focus on the analysis of the transmission phase following the creation).

- Each node generates information at a constant (deterministic) rate, indicated as $\lambda$ (dimension: [pck/s]). Although information generation at constant rate is unrealistic, it allows to obtain closed-form expressions. For comparison, note that in [5], [6] the network communication 
model is such that a node generates information only after reserving a route, i.e., it is not equipped with buffers.

- We consider a network communication scenario where only a limited number of multi-hop routes are active. We refer to the corresponding medium access control (MAC) protocol as "fixed number of (active) routes" (FNR) MAC protocol.

A simple conceptual model to visualize on-going network communications is that of associating each node with a glass, where water (corresponding to the generated information) is poured in. Whenever a node transmits to another node, the equivalent glass empties-one can imagine that there is a tube connecting the source glass with the destination glass, and water flows from the former to the latter. According to the glass-based analogy, the two possible scenarios for network communication, without and with INI, respectively, can be pictured as follows.

- Ideal (no INI) communication: in this case, all tubes connecting pairs of glasses are separated, so that the fluids do not mix.

- Realistic (INI) communication: in this case, there is only one common tube connecting all glasses, so that the fluids coming out of the glasses can mix.

A relay node forwards information generated at the source node, but in the meantime it keeps on generating information on its own. This is equivalent to saying that non-emptying glasses keep on accumulating water.

\section{Communication-Theoretic Preliminaries}

We assume that $N$ nodes are placed uniformly, at the vertices of a square grid, inside a circular area $A$. Denoting by $\rho_{S} \triangleq N / A$ the node spatial density, the distance between two neighboring nodes, $r_{L}$, can be written as $r_{L} \approx 1 / \sqrt{\rho_{S}}$. It is possible to show that each route is constituted, on average, by $\bar{n}_{h}=\lfloor\sqrt{N / \pi}\rceil$ hops, where the notation $\lfloor *\rceil$ indicates the integer value closest to $*$ [5], [6]. There can thus be, at most, $N_{R} \triangleq N / \bar{n}_{h}=\lfloor\sqrt{N \pi}\rceil$ disjoint routes simultaneously active in the network. In other words, one can imagine that at most $N_{R}$ glasses are simultaneously emptying in each RCUI. In general, we indicate by $\eta \leq N_{R}$ the number of active routes. Given that $\eta$ multi-hop routes are simultaneously active, a node has to wait $N / \eta$ RCUIs before being able to activate its own communication route. This is based on the assumption of "ideally fair" multiple access, where all nodes use the common radio channel with the same level of intensity. ${ }^{1}$

Within a RCUI, a node has to transmit the information accumulated in the previous $(N / \eta-1)$ RCUIs and the information which is being generated in the current RCUI. Hence, a node has to transmit the information which has been generated at rate $\lambda L$ during $N / \eta$ RCUIs of duration $T_{R C U I}$. Indicating by $k$ the amount of bits to be transmitted, it follows

\footnotetext{
${ }^{1}$ This assumption is motivated by the fact that the nodes are at the same hierarchical level; therefore, they have the same transmission needs.
}

that $k=\left\lfloor\lambda L T_{R C U I} N / \eta\right\rceil$. Therefore, the ECUI has duration

$$
T_{E C U I}=\frac{k}{R_{b}}=\frac{\lambda L}{R_{b}} \frac{N}{\eta} T_{R C U I} .
$$

Note that $k$ can be interpreted as the minimum necessary dimension of a node buffer. The message with $k$ bits has to be transmitted within a RCUI, i.e., the condition $T_{R C U I} \geq$ $T_{E C U I}$ has to be satisfied. This condition can be equivalently expressed as ${ }^{2}$

$$
\frac{R_{b}}{\lambda L} \geq \frac{N}{\eta}
$$

In the absence of ideal fairness, a violation of condition (2) would not allow a node to transmit, in a RCUI, all the information previously accumulated. In order to transmit all the information, the duration of the RCUI should increase, but this increase, unfortunately, would lead to a steady increase of the buffered message and then again to an increase of the duration of the RCUI, and so on. In other words, the network would become unstable. In fact, either the delay becomes unacceptable (provided that the buffer at each node has infinite dimension) or, in order to keep the delay fixed, some packets need to be discarded (if the dimension of the buffer is finite). Note also that the lower the number $\eta$ of active routes, the larger the delay a node can access the channel with and the larger the dimension of the message accumulated in the buffer. In this sense, the results presented in this paper can be considered as corresponding to an average steady-state network communication scenario.

The average sustainable number of hops, indicated by $\bar{n}_{s h}$ and introduced in [5], [6] to characterize the level of connectivity of a circuit-switched wireless sensor network, is defined as follows:

$$
\bar{n}_{s h} \triangleq \min \left\{\left\lfloor\frac{\ln \left(1-\mathrm{BER}^{\max }\right)}{\ln \left(1-\mathrm{BER}_{L}\right)}\right\rfloor,\left\lfloor\sqrt{\frac{N}{\pi}}\right\rfloor\right\}
$$

where $\mathrm{BER}^{\max }$ is the maximum acceptable $\mathrm{BER}$ at the end of a multi-hop communication route and $\mathrm{BER}_{L}$ is the link BER in the multi-hop route-we are implicitly assuming that each link experiences the same BER, but this value could change depending on the geometry of the nodes and the position of the link in the network. In other words, the average sustainable number of hops is the minimum between the maximum number of sustainable hops (which corresponds to $\left.\left\lfloor\ln \left(1-\mathrm{BER}^{\mathrm{max}}\right) / \ln \left(1-\mathrm{BER}_{L}\right)\right\rceil\right)$ and the average number of hops $\bar{n}_{h}=\lfloor\sqrt{N / \pi}\rceil$. Recalling that the hop length is $r_{L} \approx 1 / \sqrt{\rho_{S}}$, the average length $\bar{r}_{P A T H}$ of a route in the network can be written as $\bar{r}_{P A T H} \approx \bar{n}_{s h} / \sqrt{\rho_{S}}$. Since $k$ bits are transmitted in $T_{R C U I}$ seconds, it is easy to show that the effective transport capacity associated with a single route, as defined in [5], [6], can be written as

$$
C_{T, e}^{(s r)}=\frac{k}{T_{R C U I}} \bar{r}_{P A T H}=\lambda L \frac{\sqrt{N A}}{\eta} \bar{n}_{s h} .
$$

${ }^{2}$ Condition (2) can also be rewritten as $R_{b} \eta \geq N \lambda L$, i.e., by requiring that the overall generated information (i.e., $N \lambda L$ ) is lower than the maximum information flow supported by the network (i.e., $R_{b} \eta$ ). 
The aggregate effective transport capacity is obtained by adding the contributions relative to the various active routes. However, because of the possible inter-node interference (INI), it is necessary to distinguish between ideal and realistic cases. The formulas relative to the single-route effective transport capacity are formally the same in both cases, the difference being in the link SNR, indicated by $\mathrm{SNR}_{L}$, on which the link BER depends. In the rest of the paper, we will consider uncoded binary phase shift keying ${ }^{3}$ (BPSK), in which case the relationship between the link BER and the link SNR is given by

$$
\mathrm{BER}_{L}=Q\left(\sqrt{2 \mathrm{SNR}_{L}}\right)
$$

where $Q(x) \triangleq(1 / 2 \pi) \int_{x}^{\infty} e^{-r^{2} / 2} d r$. The link SNR can be written, in ideal and realistic cases, respectively, as follows:

$$
\begin{aligned}
\mathrm{SNR}_{L}^{\text {ideal }} & =\frac{P_{\text {signal }}}{P_{\text {thermal }}} \\
\mathrm{SNR}_{L}^{I N I} & =\frac{P_{\text {signal }}}{P_{\text {thermal }}+P_{I N T}}
\end{aligned}
$$

where $P_{\text {signal }}$ is the received signal power at the ending node of a link, $P_{\text {thermal }}$ is the thermal noise at the receiver side, and $P_{I N T}$ is the interference power. Assuming that the transmitted signal is affected by free space propagation loss, the received signal power can be written, according to the Friis free space formula [8], as $P_{\text {signal }}=\alpha P_{t} \rho_{S}$, with

$$
\alpha \triangleq \frac{G_{t} G_{r} c^{2}}{(4 \pi)^{2} f_{l} f_{c}^{2}}
$$

where: $G_{t}$ and $G_{r}$ are the transmitter and receiver antenna gains, respectively; $c$ is the speed of light; $f_{l}$ is a loss factor; and $f_{c}$ is the carrier frequency. The thermal noise power can be written as $P_{\text {thermal }}=F k T_{0} B$ where $F$ is the noise figure, $k \approx 1.38 \times 10^{-23} \mathrm{~J} / \mathrm{K}$ is the Boltzmann's constant, $T_{0} \approx 300 \mathrm{~K}$ is the room temperature and $B$ is the transmission bandwidth. Note that in the case of BPSK signaling $B \approx R_{b}$.

\section{IDEAL CASE}

Assuming that the maximum number (i.e., $N_{R}$ ) of independent routes are simultaneously active, the effective aggregate transport capacity is simply obtained by adding the contributions of all routes:

$$
C_{T, e}^{(i d e a l)}=N_{R} C_{T, e}^{(s r)}=\lfloor\sqrt{N \pi}\rceil C_{T, e}^{(s r)} .
$$

Since in this case disjoint multi-hop routes do not interfere $\left(P_{I N T}=0\right)$, it is immediate to recognize that for each packet generation rate the highest value of the effective transport capacity is obtained for $T_{R C U I}=T_{E C U I}$, corresponding to the case in which a node transmits during the entire interval in which the route is held. According to (1) and owing to the fact that $\eta=N_{R}$, the last condition can be equivalently rewritten as $\lambda L N=N R_{b}$.

\footnotetext{
${ }^{3}$ Note that the analysis conducted in the following can be straightforwardly generalized to other types of modulation and coding schemes.
}

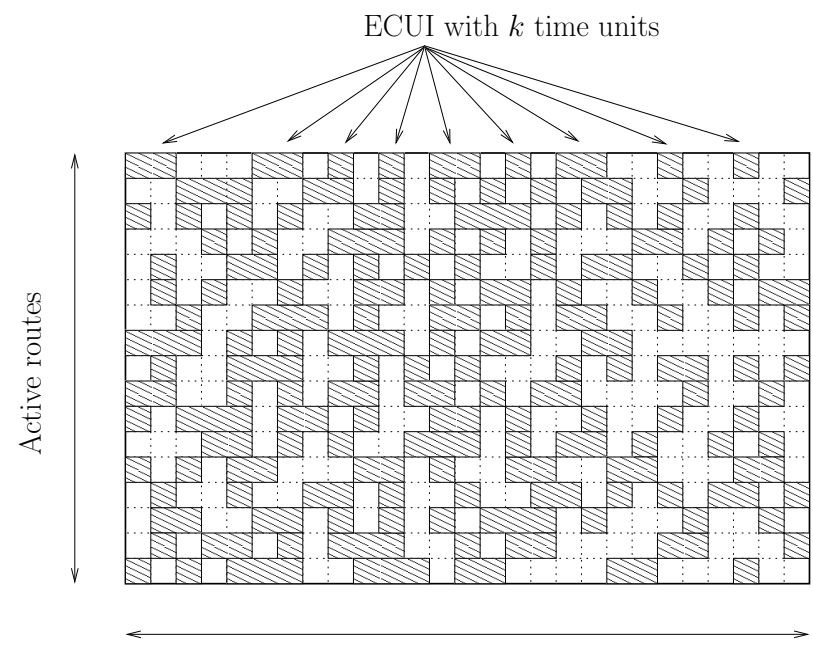

RCUI with $n$ time units

Fig. 1. $\quad N_{R}$ active routes transmitting in a RCUI with $n$ time units. Along each route $k$ bits are transmitted randomly over $n$ time units, i.e., there are gaps between successive groups of transmitted bits. In the figure, $n=27$ and $k=13$.

\section{ReAlistic CASE}

In the following, we relax the assumption that there is no INI, and we consider a more realistic network communication scenario where the active routes are interfering with each other. In particular, the effective transport capacity, when using the FNR MAC protocol, is evaluated for different values of the number $\eta$ of active routes.

\section{A. FNR MAC Protocol with $\eta=N_{R}$ Active Routes}

In this case, the number of disjoint routes simultaneously active corresponds to the maximum possible value $N_{R}$. Assuming that the basic time unit is the bit duration $1 / R_{b}$, it is possible to associate a RCUI to $n \triangleq\left\lfloor T_{R C U I} R_{b}\right\rceil$ time units. Each source node transmits for $T_{E C U I}$ seconds inside the RCUI, i.e., the message generated at each node is $k=$ $T_{E C U I} R_{b}$ time units long. For the sake of analytical simplicity, we assume that RCUIs relative to simultaneously active multihop routes start at the same instants, i.e., the active nodes are synchronized. This is clearly unrealistic, however, due to the randomized transmission inside each RCUI, this assumption can be relaxed, considering also unsynchronized transmissions in different multi-hop routes. The same conclusions hold, provided that in each route the duration of the RCUI, i.e., $T_{R C U I}$, is the same.

1) Probability of Bit Interference: Whenever a source node starts transmitting in a previously created communication route, we assume that the transmission of the $k$ bits of the message is discontinuous (or random-like), i.e, there are "gaps" between following subgroups of bits which are transmitted. This scenario can be easily depicted through the matrix model shown in Fig. 1, where each row corresponds to an active route and a filled column position indicates (on a time scale) a bit transmission. A randomized transmission inside a RCUI corresponds to an experiment where $k$ bit positions, among $n$ possible positions, are chosen. It is possible to show that 
the probability of bit interference from another single active route is independent of the considered bit position and can be written as follows:

$$
p_{I}^{\left(N_{R}\right)}=\frac{\left(\begin{array}{c}
n-1 \\
k-1
\end{array}\right)}{\left(\begin{array}{c}
n \\
k
\end{array}\right)}=\frac{k}{n}=\frac{T_{E C U I}}{T_{R C U I}}=\frac{\lambda L}{R_{b}} \frac{N}{N_{R}} .
$$

The case of bit interference in the row of interest by $m \in$ $\left\{1, \ldots, N_{R}-1\right\}$ routes corresponds, in the matrix model, to the case where there is superposition, in the corresponding bit position, with $m$ rows and no superposition with the remaining rows. Hence, the probability of interference from $m$ routes on a single bit position, indicated by $\operatorname{Pr}_{I}(m)$, is independent of the bit position and has the following binomial distribution with parameter $p_{I}^{\left(N_{R}\right)}$ :

$\operatorname{Pr}_{I}(m)=\left(\begin{array}{c}N_{R}-1 \\ m\end{array}\right)\left(p_{I}^{\left(N_{R}\right)}\right)^{m}\left(1-p_{I}^{\left(N_{R}\right)}\right)^{N_{R}-1-m} \cdot(11)$

2) Average Interference Power: As considered before, whenever there is superposition, in the same bit position, between two different routes (i.e., whenever the row corresponding to the interfering route in the matrix model and the row corresponding to the reference route have the same column position filled), there is simultaneous transmission of a bit along the two routes. Recalling the assumption of square grid uniform network topology - the conclusions hold qualitatively for any uniform network topology, i.e., not necessarily square - and assuming that (i) the link under analysis is in the center of the network (ii) all the nodes of an interfering route are simultaneously active, ${ }^{4}$ geometric considerations (omitted for lack of space), allow to derive the following expression for the average interference power generated by a single route:

$$
\bar{P}_{I N T}^{(s r)}=\frac{\alpha P_{t} \rho_{S}}{\lfloor\sqrt{N} / 2\rceil} \sum_{i=1}^{\lfloor\sqrt{N} / 2\rceil}\left(\frac{1}{i^{2}}+2 \sum_{j=1}^{\left\lfloor\frac{\bar{n}_{h}}{2}\right\rceil} \frac{1}{i^{2}+j^{2}}\right) .
$$

Under the hypothesis of $m$ interfering active routes, we simply assume that the interference power ${ }^{5}$ is $m \bar{P}_{I N T}^{(s r)}$. Recalling expression (11) for the probability $\operatorname{Pr}_{I}(m)$ of interference from $m$ other routes, the average interference power $\bar{P}_{I N T}$ can be written as

$$
\bar{P}_{I N T}=\bar{P}_{I N T}^{(s r)}\left(N_{R}-1\right) p_{I}^{\left(N_{R}\right)} .
$$

The average interference power $\bar{P}_{I N T}$ in (13) can be used in the expression for the link SNR and then to evaluate $\bar{n}_{s h}$ for the expression of the effective transport capacity. Note that the expression for the effective transport capacity with $N_{R}$ active routes is formally the same as the no INI case, the only difference being the expression for the link SNR.

\footnotetext{
${ }^{4}$ This is a very pessimistic assumption. The results obtained in the following should thus be interpreted as relative to a worst case transmission scenario.

${ }^{5}$ The expression of the interference power due to $m$ routes might be incorrect for some spatial portions of the active routes. However, if the active routes are uniformly distributed in the network, this expression should be a meaningful estimate of the interference power.
}

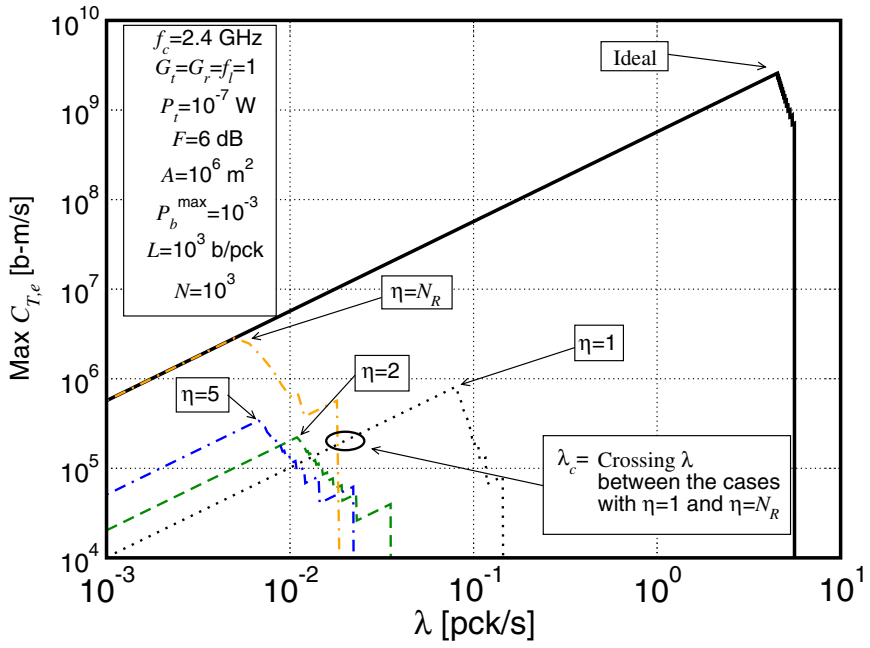

Fig. 2. Maximum effective transport capacity versus packet generation rate $\lambda$. Comparison between a realistic case (with FNR MAC protocol and various values of the number of active routes $\eta$ ) and the ideal (no INI) case.

\section{B. General Case with $\eta$ Active Routes}

The previous analysis with $N_{R}$ active routes can be extended naturally to a general case with $\eta \leq N_{R}$ active routesin other words, the network communication protocol is such that $\eta$ routes are simultaneously active in each RCUI. Hence, (10), (11) and (13) can be systematically extended to this case by replacing $N_{R}$ with $\eta$.

\section{RESULTS AND DISCUSSION}

The maximum achievable effective transport capacity is shown, as a function of the packet generation rate $\lambda$, in Fig. 2. In particular, the curves relative to FNR MAC protocols with $\eta=1,3,5$, and $N_{R}=\left\lfloor\sqrt{\pi 10^{3}}\right\rceil=56$ active routes are shown. For comparison, the curve (thick solid line) relative to the ideal case without INI is also shown. The peak point of each curve is indicated explicitly in the figure. It is immediate to recognize that there exists a critical packet generation rate, indicated by $\lambda_{c}\left(\lambda_{c} \approx 0.018 \mathrm{pck} / \mathrm{s}\right.$ for the values considered in Fig. 2), below which the effective transport capacity is maximized by activating the largest possible number of multi-hop routes. For values of the packet generation rate larger than this critical value, the highest effective transport capacity is obtained by activating only a single route in the network. Note that even if in the case of FNR with $\eta=1$ the interference power is $P_{I N T}=0$, the packet generation rate corresponding to the peak is lower than that in the case with the maximum number $\eta=N_{R}$ of active routes. This is due to condition (2), which becomes very stringent for $\eta=1$ : in other words, the datarate (i.e., the bandwidth) needs to be very large, so that the effect of the thermal noise becomes significant even at lower packet generation rates. The two extreme cases of FNR MAC protocol with $\eta=N_{R}$ and $\eta=1$ active routes, respectively, are very similar to the two MAC protocols proposed in [5], [6] and outlined in the following.

- The first MAC protocol is such that each node, after 


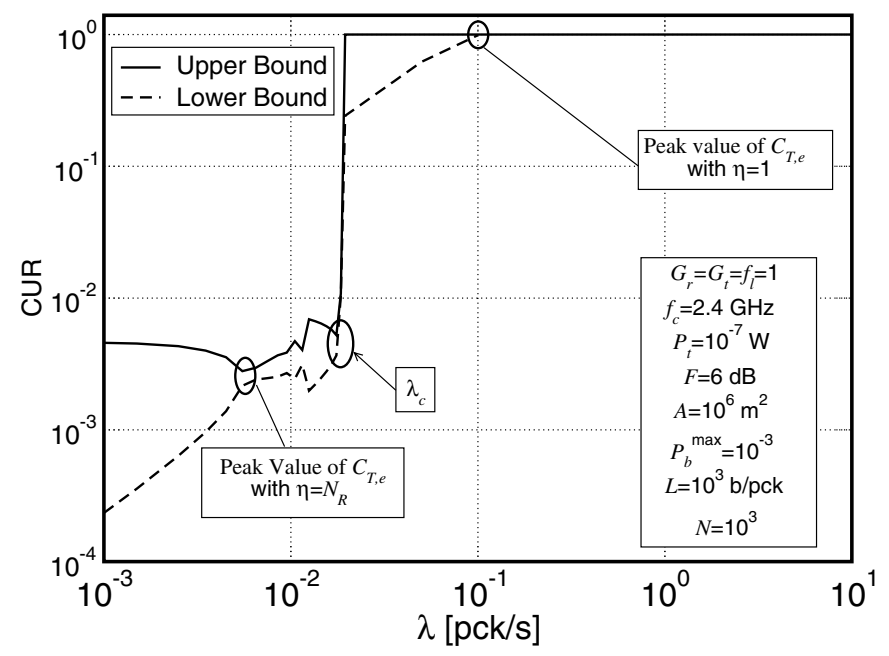

Fig. 3. Upper and lower bounds for the channel utilization.

reserving a route, transmits without sensing the channel. ${ }^{6}$

- The second MAC protocol is characterized by the fact that a node, after reserving a route, senses the channel before transmitting: if no transmission is going on, then the node starts transmitting. ${ }^{7}$

Extending the approach proposed in [6] for the analysis of the connectivity of a circuit-switched ad hoc wireless network, it is possible to show that, in the case with FNR MAC protocol with $\eta=N_{R}$ active routes, for values of the packet generation rate larger than that corresponding to the peak value, full connectivity is lost. In other words, even if the numerical value of the effective transport capacity with $N_{R}$ active routes is larger than in the case with a lower number of active routes, in the latter case connectivity might still be preserved. Due to lack of space, we do not comment further on this aspect.

The effective transport capacity results shown in Fig. 2, are obtained by optimizing, for each value of $\lambda$, the data-rate $R_{b}$. It is possible to show that for each packet generation rate $\lambda$, there exists a data-rate range $\left(R_{b}^{\min }, R_{b}^{\min }+\Delta R_{b}\right)$ where the effective transport capacity is constant and maximum (as it is shown in [6] for a time-continuous network communication scenario with Poisson packet generation). Hence, the CUR can be bounded as follows:

$$
\frac{\lambda L}{R_{b}^{\text {min }}+\Delta R_{b}} \frac{N}{\eta} \leq \mathrm{CUR} \leq \frac{\lambda L}{R_{b}^{\min }} \frac{N}{\eta} .
$$

The upper and lower bounds for the CUR, are shown, as a function of the packet generation rate $\lambda$ in Fig. 3. For each value of $\lambda$, the number of active routes is optimized. More precisely, as indicated in Fig. 3, for values of $\lambda$ lower than $\lambda_{c}$,

\footnotetext{
${ }^{6}$ This MAC protocol was referred in [5], [6] as Aloha MAC protocol, for its resemblance, in terms of route activation independent from the activity of other nodes in the network, with the classical Aloha MAC protocol [9]. However, there are significant differences which make the proposed protocol different from the classical Aloha MAC protocol: (i) multi-hop route reservation and (ii) no use of retransmission techniques.

${ }^{7}$ This MAC protocol was referred in [5], [6] as per-route carrier sense multiple access (PR-CSMA) MAC protocol, for its resemblance, in terms of route activation after sensing, with the classic CSMA MAC protocol [10].
}

the upper and lower bounds refer to a network communication scenario with $\eta=N_{R}$ active routes. Note that there is a sort of bottleneck between the upper and lower bounds for the value of the packet generation rate corresponding to the peak of the effective transport capacity with $\eta=N_{R}$ active routes. In other words, the maximum effective transport capacity is obtained for a precise (and very low) value of the CUR. This has important consequences in terms of delay performance, and this aspect is currently under investigation.

The obtained results quantify an important (and intuitive) trade-off in circuit-switched ad hoc wireless networks, between number of routes simultaneously active and the CUR. More precisely, the larger the number of nodes which are simultaneously transmitting, the lower the CUR has to be.

\section{CONCLUDING REMARKS}

Based on an equivalent discrete-time network communication model, the performance of circuit-switched wireless sensor networks with various numbers of active routes has been evaluated in terms of (i) effective transport capacity and (ii) CUR. While in terms of effective transport capacity it has been shown that a FNR MAC protocol with the maximum number of active routes guarantees no loss with respect to an ideal (no INI) scheme for very low packet generation rate, the price to be paid is a very small CUR. For larger packet generation rates, the best choice is activating a single route in the network, in which case the CUR is equal to one. A more accurate analysis of the relationship between effective transport capacity and CUR is currently under investigation, in particular in the case where transmission inside a RCUI is not randomized.

\section{REFERENCES}

[1] C. E. Perkins, Ad hoc Networking. Upper Saddle River, NJ, U.S.A.: Addison-Wesley, 2001.

[2] C.-K. Toh, Ad hoc Mobile Wireless Networks. Upper Saddle River, NJ, U.S.A.: Prentice-Hall, 2002.

[3] O. K. Tonguz and G. Ferrari, "A Communication-Theoretic Framework for Ad Hoc Wireless Networks," Carnegie Mellon University, ECE Dept., Tech. Rep., February 2003.

[4] - "Extensions of A Communication-Theoretic Framework for Ad Hoc Wireless Networks," Carnegie Mellon University, ECE Dept., Tech. Rep., September 2003.

[5] G. Ferrari and O. K. Tonguz, "Performance of circuit-switched ad hoc wireless networks with Aloha and PR-CSMA MAC protocols," in Proc. IEEE Global Telecommun. Conf. (GLOBECOM), San Francisco, USA, December 2003, pp. $2824-2829$.

[6] _ "MAC protocols and transport capacity in ad hoc wireless networks: Aloha versus PR-CSMA," in Proc. IEEE Military Comm. Conf. (MILCOM), Boston, USA, October 2003.

[7] P. Gupta and P. R. Kumar, "The capacity of wireless networks," IEEE Trans. Inform. Theory, vol. 46, pp. 388-404, March 2000.

[8] T. S. Rappaport, Wireless Communications. Principles \& Practice. Upper Saddle River, NJ, U.S.A.: Prentice-Hall, 2002, second edition.

[9] N. Abramson, "The throughput of packet broadcasting channels," IEEE Trans. Commun., vol. 25, pp. 117-128, January 1977.

[10] L. Kleinrock and F. A. Tobagi, "Packet switching in radio channels: Part I-Carrier sense multiple-access modes and their throughput-delay characteristics," IEEE Trans. Commun., vol. 23, no. 12, pp. 1400-1416, December 1975. 